\title{
String Gauge Symmetries in the Conformally Gauge-Fixed Polyakov D1 Brane Action in the Presence of Background Gauge Fields*
}

\author{
Usha Kulshreshtha ${ }^{1}$, Daya Shankar Kulshreshtha ${ }^{2}$ \\ ${ }^{1}$ Department of Physics, Kirori Mal College, University of Delhi, Delhi, India \\ ${ }^{2}$ Department of Physics and Astrophysics, University of Delhi, Delhi, India \\ Email: \{ushakulsh, dskulsh\}@gmail.com
}

Received September 5, 2011; revised November 6, 2011; accepted December 1, 2011

\begin{abstract}
Recently we have studied the instant-form quantization (IFQ) and the light-front quantization (LFQ) of the conformally gauge-fixed Polyakov $D 1$ brane action using the Hamiltonian and path integral formulations. The IFQ is studied in the equal world-sheet time framework on the hyperplanes defined by the world- sheet time $\sigma^{0}=\tau=$ constant and the LFQ in the equal light-cone world-sheet time framework, on the hyperplanes of the light-front defined by the light-cone world-sheet time $\sigma^{+}=(\tau+\sigma)=$ constant. The light-front theory is seen to be a constrained system in the sense of Dirac in contrast to the instant-form theory. However, owing to the gauge anomalous nature of these theories, both of these theories are seen to lack the usual string gauge symmetries defined by the world-sheet reparametrization invariance (WSRI) and the Weyl invariance (WI). In the present work we show that these theories when considered in the presence of background gauge fields such as the NSNS 2-form gauge field $B_{\alpha \beta}(\sigma, \tau)$ or in the presence of $U(1)$ gauge field $A_{\alpha}(\sigma, \tau)$ and the constant scalar axion field $C(\sigma, \tau)$, then they are seen to possess the usual string gauge symmetries (WSRI and WI). In fact, these background gauge fields are seen to behave as the Wess-Zumino/Stueckelberg fields and the terms containing these fields are seen to behave as Wess-Zumino or Stueckelberg terms for these theories.
\end{abstract}

Keywords: Light-Front Quantization; Hamiltonian Quantization; Path Integral Quantization; Constrained Dynamics; Constraint Quantization; Gauge Symmetry; String Gauge Symmetry; String Theory; D1-Brane Actions; Polyakov Action; Light-Cone Quantization

Study of D-brane actions [1-19] is a domain of wider interest in string theories. Polyakov action does not involve any square root and is in particular, simpler to study. Recently, we have studied IFQ of this action [12] for the $D 1$ brane in the conformal gauge (CG), using the Hamiltonian [20] and path integral [21-25] formulations in the instant-form (IF) of dynamics (on the hyperplanes defined by the world-sheet (WS) time $\sigma^{0}=\tau=$ constant ) $[26,27]$. We have also studied its LFQ [13-19] using the light-front (LF) dynamics (on the hyperplanes of the LF defined by the light-cone (LC) WS time $\sigma^{+}=(\tau+\sigma)=$ constant ) [13-19,26-32].

The LF theory [13-19] is seen to be a constrained system in the sense of Dirac [20], which is in contrast to the corresponding IF theory [12], where the theory remains unconstrained in the sense of Dirac. The LF theory is

*Part of this work was presented as an Invited Contributed Talk by DSK at the International Light-Cone Conference LC2010: Relativistic Hadronic and Particle Physics, Valencia, Spain, June 14-18, 2010. seen to possess a set of twenty six second-class contraints [13-19]. Further, the conformally gauge-fixed Polyakov $D 1$ brane action (CGFPD1BA) describing a gauge-noninvariant (GNI) theory (being a gauge-fixed theory) is seen to describe a gauge-invariant (GI) theory in the presence of an antisymmetric NSNS 2-form gauge field $B_{\alpha \beta}(\tau, \sigma)$ [13].

Recently we have shown [13] that this NSNS 2-form gauge field behaves like a Wess-Zumino (WZ) field and the term involving this field behaves like a WZ term for the CGFPD1BA [13]. We have also studied the Hamiltonian [20] and path integral [21-25] formulations of the CGFPD1BA with and without a scalar dilaton field in the IF [12] as well as in the LF [13-19] dynamics. In both the above cases the theory is seen (as expected) to be gaugenoninvariant (GNI), possessing a set of second-class constraints in each case, owing to the conformal gaugefixing [1-8,12-19] of the theory.

The CGFPD1BA being GNI does not respect the usual 
(string) gauge symmetries defined by the WS reparametrization invariance (WSRI) and the Weyl invariance (WI). However, in the presence of a constant 2-form gauge field $B_{\alpha \beta}$ it is seen [13] to describe a gauge-inavriant (GI) theory respecting the usual string gauge symmetries defined by the WSRI and the WI [13].

The IF and LF Hamiltonian and path integral formulations of this theory in the presence of the constant 2-form gauge field $B_{\alpha \beta}$ have been studied by us in Ref. [13]. In the present work, we consider the question of the string gauge symmetries associated with the Polyakov D1 brane action in the presence of some other background fields such as the $U(1)$ gauge field $A^{\mu}(\tau, \sigma)$ and the constant scalar axion field $C(\tau, \sigma)$ [19]. The Polyakov $D 1$ brane action in a d-dimensional curved background $h_{\alpha \beta}$ is defined by $[1-8,12-19]$ :

$$
\begin{gathered}
\tilde{S}=\int L \mathrm{~d}^{2} \sigma \\
L=\left[\frac{-T}{2} \sqrt{-h} h^{\alpha \beta} G_{\alpha \beta}\right] \\
h=\operatorname{det}\left(h_{\alpha \beta}\right), G_{\alpha \beta}=\partial_{\alpha} X^{\mu} \partial_{\beta} X^{v} \eta_{\mu v} \\
\eta_{\mu v}=\operatorname{diag}(-1,+1, \cdots,+1) \\
\mu, v=0,1, i ., \quad i=2,3, \cdots,(d-1), \alpha, \beta=01(\text { IFQ })(1 \mathrm{~d}) \\
\mu, v=+,-, i ., i=2,3, \cdots,(d-1), \alpha, \beta=+,-(\operatorname{IF} Q)(1 \mathrm{f})
\end{gathered}
$$

Here $\sigma^{\alpha} \equiv(\tau, \sigma)$ are the two parameters describing the worldsheet (WS). The overdots and primes would denote the derivatives with respect to $\tau$ and $\sigma . T$ is the string tension. $G_{\alpha \beta}$ is the induced metric on the WS and $X^{\mu}(\tau, \sigma)$ are the maps of the WS into the $d$-dimensional Minkowski space and describe the strings evolution in space-time [1-16]. $h_{\alpha \beta}$ are the auxiliary fields (which turn out to be proportional to the metric tensor $\eta_{\alpha \beta}$ of the two-dimensional surface swept out by the string). One can think of $\tilde{S}$ as the action describing $d$ massless scalar fields $X^{\mu}$ in two dimensions moving on a curved background $h_{\alpha \beta}$. Also because the metric components $h_{\alpha \beta}$ are varied in above equation, the 2dimensional gravitational field $h_{\alpha \beta}$ is treated not as a given background field, but rather as an adjustable quantity coupled to the scalar fields [1-8,12-19]. The action $\tilde{S}$ has the well-known three local gauge symmetries given by the 2-dimensional WSRI and WI [1-8,12-19] as follows:

$$
\begin{gathered}
X^{\mu} \rightarrow \tilde{X}^{\mu}=\left[X^{\mu}+\delta X^{\mu}\right] \\
\delta X^{\mu}=\left[\zeta^{\alpha}\left(\partial_{\alpha} X^{\mu}\right)\right] \\
h^{\alpha \beta} \rightarrow \tilde{h}^{\alpha \beta}=\left[h^{\alpha \beta}+\delta h^{\alpha \beta}\right] \\
\delta h^{\alpha \beta}=\left[\zeta^{\gamma} \partial_{\gamma} h^{\alpha \beta}-\partial_{\gamma} \zeta^{\alpha} h^{\gamma \beta}-\partial_{\gamma} \zeta^{\beta} h^{\alpha \gamma}\right]
\end{gathered}
$$

$$
h_{\alpha \beta} \rightarrow[\Omega] h_{\alpha \beta}
$$

where the WSRI is defined for the two parameters $\zeta^{\alpha} \equiv \zeta^{\alpha}(\tau, \sigma)$; and the WI and is specified by a function $\Omega \equiv \Omega(\tau, \sigma)$. In the following we would, however, work in the so-called orthonormal gauge where one sets $\Omega=1$. Also for the CGFPD1BA one makes use of the fact that the 2-dimensional metric $h_{\alpha \beta}$ is also specified by three independent functions as it is a symmetric $2 \times 2$ metric. one can therefore use these gauge symmetries of the theory to choose $h_{\alpha \beta}$ to be of a particular form in the IFQ (on the hyperplanes defined by $x^{0}=t=$ constant) as follows:

$$
h_{\alpha \beta}:=\eta_{\alpha \beta}, h^{\alpha \beta}:=\eta^{\alpha \beta}
$$

For the IF dynamics we take [1-12]

$$
\begin{gathered}
h_{\alpha \beta}=\eta_{\alpha \beta}=\left(\begin{array}{rr}
-1 & 0 \\
0 & +1
\end{array}\right) \\
h^{\alpha \beta}=\eta^{\alpha \beta}=\left(\begin{array}{rr}
-1 & 0 \\
0 & +1
\end{array}\right)
\end{gathered}
$$

with

$$
\sqrt{-h}=\sqrt{-\operatorname{det}\left(h_{\alpha \beta}\right)}=+1
$$

In LF formulation we use the Light-Cone (LC) variables defined by:

$$
\sigma^{ \pm}:=(\tau \pm \sigma) ; \quad X^{ \pm}:=\left(X^{0} \pm X^{1}\right) / \sqrt{2}
$$

In this case for the LFQ (on the hyperplanes defined by $x^{+}=\tau=$ constant) we take:

$$
\begin{aligned}
& h_{\alpha \beta}:=\eta_{\alpha \beta}=\left(\begin{array}{cc}
0 & -1 / 2 \\
-1 / 2 & 0
\end{array}\right) \\
& h^{\alpha \beta}:=\eta^{\alpha \beta}=\left(\begin{array}{rr}
0 & -2 \\
-2 & 0
\end{array}\right)
\end{aligned}
$$

with

$$
\sqrt{-h}=\sqrt{-\operatorname{det}\left(h_{\alpha \beta}\right)}=+1 / 2
$$

Now the action $\tilde{S}$ in the CG (in the IF and LF) finally reads[1-3,9-16]:

$$
\begin{gathered}
S^{N}=\int L^{N} \mathrm{~d}^{2} \sigma \\
L^{N}=\left(\frac{-T}{2}\right)\left[\partial^{\beta} X^{\mu} \partial_{\beta} X_{\mu}\right] \\
\beta=0,1 ; \quad \mu=0,1, i ; \quad i=2,3, \cdots, 25 \text { (IFQ) } \\
\beta=+,-; \quad \mu=+,-, i ; \quad i=2,3, \cdots, 25 \text { (LFQ) }
\end{gathered}
$$

The action $S^{N}$ is the CGFPD1BA. The above action in IFQ [12] is seen to be an unconstrained system and in LFQ [13,14] it is seen to possess a set of 26 second-class constraints [13] implying that the corresponding theories 
are seen to be gauge anomalous and GNI and therefore they do not possess the local gauge symmetries defined by the WSRI and the WI. The Hamiltonian and path integral formulations of this CGFPD1BA defined by the action $S^{N}$ have been studied by us Ref. [12]. When this above action is considered in the presence of a Scalar Dilaton field in the IFQ as well as in the LFQ then also it is seen to possess sets of second-class constraints implying that it remains GNI theory which does not respect the string gauge symmetries: the WSRI and the WI [1-8,12-19].

This action is thus seen to lack the local gauge symmetries. This is in contrast to the fact that the original action $\tilde{S}$ had the local gauge symmetries and was therefore GI. The theory defined by the action $S^{N}$, on the other hand describe GNI. This is not surprising at all because the theory defined by $S^{N}$ is afterall (conformally) gauge-fixed theory and consequently not expected to be GI anyway. Infact, the IF theory defined by $S^{N}$ is seen to be unconstrained [12] whereas the LF theory is seen to possess a set of 26 second-class constraints [13-19]. In both the cases theory does not respect the usual local string gauge symmetries defined by WSRI and WI.

We now consider this CGFPD1BA in the presence of a constant background antisymmetric 2-form gauge field $B_{\alpha \beta}$ studied earlier by Schmidhuber, de Alwis and Sato, Tseytlin and Abou Zeid and Hull and others defined by [1-8,13]:

$$
\begin{gathered}
S^{I}=\int L^{I} \mathrm{~d}^{2} \sigma, L^{I}=\left[L^{C}+L^{B}\right] \\
L^{C}=\left[\lambda L^{N}\right]=\left(\frac{-T}{2}\right)\left[\lambda \partial^{\beta} X^{\mu} \partial_{\beta} X_{\mu}\right] \\
L^{B}=\left(\frac{-T}{2}\right)\left[\Lambda \varepsilon^{\alpha \beta} B_{\alpha \beta}\right] \\
\lambda=\sqrt{\left(1+\Lambda^{2}\right)}, \Lambda=\text { constant, } \varepsilon^{\alpha \beta}=\left(\begin{array}{cc}
0 & 1 \\
-1 & 0
\end{array}\right) \\
B_{\alpha \beta}:=\partial_{\alpha} X^{\mu} \partial_{\beta} X^{v} B_{\mu v}, B_{\alpha \beta}=\left(\begin{array}{cc}
0 & B \\
-B & 0
\end{array}\right) \\
B=B_{01}=-B_{10}(\mathrm{IFQ}) \\
B=B_{+-}=-B_{-+}(\mathrm{LFQ}) \\
\alpha, \beta=0,1, \quad \mu=0,1, i, \quad i=2,3, \cdots, 25(\mathrm{IFQ}) \\
\alpha, \beta=+,-, \quad \mu=+,-, i, \quad i=2,3, \cdots, 25(\mathrm{LFQ})
\end{gathered}
$$

The above action is seen to possess (only) one firstclass constraint in IFQ and a set of 27 first-class constraints in LFQ [13-19]. Accordingly the theory in both the cases is seen to three local gauge symmetries given by the two-dimensional WSRI and the WI:

$$
X^{\mu} \rightarrow \tilde{X}^{\mu}=\left[X^{\mu}+\delta X^{\mu}\right]
$$

$$
\begin{gathered}
\delta X^{\mu}=\left[\zeta^{\alpha}\left(\partial_{\alpha} X^{\mu}\right)\right] \\
h^{\alpha \beta} \rightarrow \tilde{h}^{\alpha \beta}=\left[h^{\alpha \beta}+\delta h^{\alpha \beta}\right] \\
\delta h^{\alpha \beta}=\left[\zeta^{\gamma} \partial_{\gamma} h^{\alpha \beta}-\partial_{\gamma} \zeta^{\alpha} h^{\gamma \beta}-\partial_{\gamma} \zeta^{\beta} h^{\alpha \gamma}\right] \\
B_{\alpha \beta} \rightarrow \widetilde{B}_{\alpha \beta}=\left[B_{\alpha \beta}+\delta B_{\alpha \beta}\right] \\
\delta B_{\alpha \beta}=\left[\zeta^{\alpha} \partial_{\alpha} B_{\alpha \beta}\right] \\
h_{\alpha \beta} \rightarrow[\Omega] h_{\alpha \beta}
\end{gathered}
$$

It is important to recollect here that the 2-form gauge field $B_{\alpha \beta}$ is a scalar field in the target-space whereas it is a constant anti-symmetric tensor field in the worldheet space. In Ref. [13], we have studied the Hamiltonian and path integral formulations of this theory under the gauge $B \approx 0 \quad$ [13].

In this work we investigate the string gauge symmetries of CGFPD1BA describing a GNI theory in the presence of a $U(1)$ gauge field $A_{\alpha}\left(\equiv A_{\alpha}(\tau, \sigma)\right)$ and a constant scalar axion field $C(\equiv C(\tau, \sigma))$ [19] and show that the CGFPD1BA describing a GNI theory (being a gauge-fixed theory) is seen to describe a GI theory when considered in the presence of above background fields.

We also show that the $U(1)$ gauge field $A_{\alpha}(\tau, \sigma)$ and the constant scalar axion field $C(\tau, \sigma)$ are both seen to behave like the Wess-Zumino (WZ) fields [19] and the term involving these fields is seen to behave like a WZ term for the CGFPD1BA [19]. Here the field $A_{\alpha}$ is a scalar field in the target space and a vector field in the WS space and the axion field $C$ is a constant scalar field in both the target space as well as in the WS space [19].

We find that the resulting theory obtained in the above manner describes a GI system respecting the usual string gauge symmetries defined by the WSRI and the WI. It is seen that the axion field $C$ and the $U(1)$ gauge field $A_{\alpha}$, in the resulting theory behave like the WZ fields and the term involving these fields behaves like a WZ term for the CGFPD1BA [19].

The situation in the present case is seen to be exactly analogous to a theory where one considers the CGFPD1BA in the presence of a 2-form gauge field $B_{\alpha B}$ as studied by us in Ref. [13], where the field $B_{\alpha \beta}$ behaves like a WZ field and the term involving this field behaves like a WZ term for the CGFPD1BA [13].

The CGFPD1BA in the presence of a constant background scalar axion field $C$ and an $U(1)$ gauge field $A_{\alpha}$ is defined by $[1-8,19]$ :

$$
\begin{gathered}
S^{I}=\int L^{I} \mathrm{~d}^{2} \sigma, L^{I}=\left(L^{C}+L^{A}\right) \\
L^{C}=\left(\lambda L^{N}\right)=\left(\frac{-T}{2}\right)\left(\lambda \partial^{\beta} X^{\mu} \partial_{\beta} X_{\mu}\right)
\end{gathered}
$$




$$
\begin{gathered}
L^{A}=\left(\frac{-T}{2}\right)\left(-\Lambda C \varepsilon^{\alpha \beta} F_{\alpha \beta}\right) \\
\lambda=\sqrt{\left(1+\Lambda^{2}\right)}, \Lambda=\mathrm{constant} \\
\varepsilon^{\alpha \beta}=\left(\begin{array}{rr}
0 & 1 \\
-1 & 0
\end{array}\right), F_{\alpha \beta}=\left(\partial_{\alpha} A_{\beta}-\partial_{\beta} A_{\alpha}\right) \\
f=F_{01}=-F_{01}(\mathrm{IFQ}) \\
f=F_{+-}=-F_{-+}(\mathrm{LFQ}) \\
\alpha, \beta=0,1, \quad \mu=0,1, i, \quad i=2,3, \cdots, 25 \text { (IFQ) } \\
\alpha, \beta=+,-, \quad \mu=+,-, i, \quad i=2,3, \cdots, 25(\mathrm{LFQ})
\end{gathered}
$$

In IFQ the theory is seen to possess a set of 3 firstclass constraints:

$$
\Psi_{1}=\Pi^{0} \approx 0, \Psi_{2}=(E-\Lambda T C) \approx 0, \Psi_{3}=\Pi_{c} \approx 0
$$

where $P^{\mu}, \Pi^{0}, E\left(\equiv \Pi^{1}\right)$ and $\Pi_{C}$ are the canonical momenta conjugate respectively to $X_{\mu}, A_{0}, A_{1}$ and $C$. Now the matrix of the Poission brackets of the constraints $\Psi_{i}$ is seen to be singular implying that the constraints $\Psi_{i}$ form a set of first-class constraints and that the theory described by the above action is a GI theory [13-19]. The LFQ of this theory alsorevels that the LF theory possesses a set of 29 first-class constraints:

$$
\begin{aligned}
& \chi_{1}=\Pi^{+} \approx 0 \\
& \chi_{2}=\left(\Pi^{-}-\Lambda T C\right) \approx 0 \\
& \chi_{3}=\Pi_{c} \approx 0 \\
& \chi_{4}=\left[P^{+}+\left(\frac{\lambda T}{2}\right)\left(\partial_{-} X^{+}\right)\right] \approx 0 \\
& \chi_{5}=\left[P^{-}+\left(\frac{\lambda T}{2}\right)\left(\partial_{-} X^{-}\right)\right] \approx 0 \\
& \chi_{i}=\left[P_{i}+\left(\frac{\lambda T}{2}\right)\left(\partial_{-} X^{i}\right)\right] \approx 0, i=2,3, \cdots, 25 .
\end{aligned}
$$

where $P^{+}, P^{-}, P_{i}, \Pi_{c}, \Pi^{+}$and $\Pi^{-}$are the momenta canonically conjugate respectively to $X^{-}, X^{+}$, $X_{i}, C, A^{-}$and $A^{+}$.

Accordingly the theory in both the cases seen to posses three local gauge symmetries given by the two dimensional WSRI and the WI defined by [1-19]

$$
\begin{aligned}
& X^{\mu} \rightarrow \tilde{X}^{\mu}=\left[X^{\mu}+\delta X^{\mu}\right] \\
& \delta X^{\mu}=\left[\zeta^{\alpha}\left(\partial_{\alpha} X^{\mu}\right)\right] \\
& h^{\alpha \beta} \rightarrow \tilde{h}^{\alpha \beta}=\left[h^{\alpha \beta}+\delta h^{\alpha \beta}\right]
\end{aligned}
$$

$$
\begin{gathered}
\delta h^{\alpha \beta}=\left[\zeta^{\gamma} \partial_{\gamma} h^{\alpha \beta}-\partial_{\gamma} \zeta^{\alpha} h^{\gamma \beta}-\partial_{\gamma} \zeta^{\beta} h^{\alpha \gamma}\right] \\
A_{\beta} \rightarrow \tilde{A}_{\beta}=\left[A_{\beta}+\delta A_{\beta}\right] \\
\delta A_{\beta}=\left[\zeta^{\alpha} \partial_{\alpha} A_{\beta}\right] \\
C \rightarrow \tilde{C}_{\alpha}=[C+\delta C] \\
\delta C=\left[\zeta^{\alpha} \partial_{\alpha} C\right] \\
h_{\alpha \beta} \rightarrow\left[\Omega h_{\alpha \beta}\right]
\end{gathered}
$$

The above theory is thus seen to be GI possessing the three local gauge symmetries defined by the two-dimensional WSRI and the WI in both the IF and LF dynamics.

In conclusion, the Polyakov D1 brane action in a ddimensional courved background $h_{\alpha \beta}$ defined by $\tilde{S}$ is GI and it possesses the well-known three local string gauge symmetries.

However, under conformal gauge-fixing, the CGFPD1BA is no longer GI as expected and it also does not possess the local string gauge symmetries being a gauge-fixed theory. Hovever, this GNI theory when considered in the presence of a contant background scalar axiom field $C$ and an $U(1)$ gauge field $A_{\alpha}$ it is seen to become a GI theory possessing the three local string gauge symmetries [19].

The scalar axion field $C$ and the $U(1)$ gauge field $A_{\alpha}$ are seen to behave like the WZ fields and the term $\left(L^{A}\right)$ involving these fields is seen to behave like a WZ term for the CGFPD1BA [19], which in the absence of this term is seen to possess a set of second-class constraints and consequently describes a GNI theory which does not respect the local string gauge symmetries.

The situation in the present case is analogous to a theory where one considerers the CGFPD1BA in the presence of a constant 2-form gauge field $B_{\alpha \beta}$ which behaves like a WZ field and the term involving this field behaves like a WZ term for the CGFPD1BA [13].

\section{REFERENCES}

[1] D. Luest and S. Theisen, "Lectures in String Theory," Lecture Notes in Physics, Vol. 346, 1989.

[2] L. Brink and M. Henneaux, "Principles of String Theory,” Plenum Press, New York, 1988.

[3] C. V. Johnson, "D-Brane Primer," Prepared for Theoretical Advanced Study Institute in Elementary Particle Physics (TASI 99): Strings, Branes, and Gravity, Boulder, Colorado, 1999, pp. 129-350.

[4] M. Aganagic, J. Park, C. Popescu and J. Schwarz, "Dual D-Brane Actions," Nuclear Physics, Vol. B496, No. 1-2, 1997, pp. 215-230. doi:10.1016/S0550-3213(97)00257-5

[5] M. Abou Zeid and C. M. Hull, "Intrinsic Geometry of 
D-Branes,” Physics Letters, Vol. B404, No. 3-4, 1997, pp. 264-270.

[6] C. Schmidhuber, "D-Brane Actions," Nuclear Physics, Vol. B467, No. 1-2, 1996, pp. 146-158. doi:10.1016/0550-3213(96)00092-2

[7] S. P. de Alwis and K. Sato, "D-Strings and F-Strings from String Loops,” Physical Review, Vol. D53, No. 12, 1996, pp. 7187-7196.

[8] A. A. Tseytlin, "Self Duality of Born-Infeld Action and Dirichlet Three-Brane of Type IIB Super String Theory," Nuclear Physics, Vol. B469, No. 1-2, 1996, pp. 51-67. doi:10.1016/0550-3213(96)00173-3

[9] U. kulshreshtha and D. S. Kulshreshtha, "Hamiltonian and Path Integral Formulations of the Nambu-Goto D1 Brane Action with and without a Dilaton Field under Gauge-Fixing," International Journal of Theoretical Physics, Vol. 43, No. 12, 2004, pp. 2355-2369. doi:10.1007/s10773-004-7704-5

[10] U. kulshreshtha and D. S. Kulshreshtha, "Hamiltonian and Path Integral Formulations of the Born-InfeldNambu-Goto D1 Brane Action with and without a Dilaton Field under Gauge-Fixing," International Journal of Theoretical Physics, Vol. 44, No. 5, 2005, pp. 587-603. doi:10.1007/s10773-005-3985-6

[11] U. kulshreshtha and D. S. Kulshreshtha, "Hamiltonian and Path Integral Formulations of the Dirac-Born-InfeldNambu-Goto D1 Brane Action with and without a Dilaton Field under Gauge-Fixing," European Physical Journal, Vol. C29, No. 3, 2003, pp. 453-461.

[12] U. Kulshreshtha and D. S. Kulshreshtha, "Hamiltonian and Path Integral Quantization of the Conformally GaugeFixed Polyakov D1 Brane Action in the Presence of a Scalar Dilation Field,” International Journal of Theoretical Physics, Vol. 48, No. 4, 2009, pp. 937-944. doi:10.1007/s10773-008-9866-z

[13] U. kulshreshtha and D. S. Kulshreshtha, "Conformally Gauge-Fixed Polyakov D1 Brane Action in the Presence of a 2-Form Gauge Field: The Instant-Form and FrontForm Hamiltonian and Path Integral Formulations," Physics Letters, Vol. B555, No. 3-4, 2003, pp. 255-263.

[14] D. S. Kulshreshtha, "Polyakov D1 Brane Action on the Light-Front,” Light-Cone 2008: Relativistic Nuclear and Particle Physics, Mulhouse, 7-11 July 2008.

[15] U. Kulshreshtha and D. S. Kulshreshtha, "Light-Front Hamiltonian and Path Integral Quantization of the Conformally Guage-Fixed Polyakov D1 Brane Action,” Journal of Modern Physics, Vol. 2, No. 5, 2011, pp. 335-340. doi:10.4236/jmp.2011.25041

[16] U. Kulshreshtha and D. S. Kulshreshtha, "Light-Front Hamiltonian and Path Integral Formulations of the Conformally Gauge-Fixed Polyakov D1 Brane Action in the Presence of a Scalar Dilaton Field,” Journal of Modern Physics, Vol. 2, No. 8, 2011, pp. 826-833. doi:10.4236/jmp.2011.28097

[17] D. S. Kulshreshtha, "Light-Front Quantization of the Polyakov D1 Brane Action with a Scalar Dilaton Field,”
Light-Cone 2007: Relativistic Hadronic and Nuclear Physics, Columbus, 14-18 May 2007.

[18] D. S. Kulshreshtha, "String Gauge Symmetries of the Light-Front Polyakov D1 Brane Action,” Light-Cone International Workshop on Relativistic Hadronic and Particle Physics, Valencia, 14-18 June 2010.

[19] D. S. Kulshreshtha, "Light-Front Quantization of Conformally Gauge-Fixed Polyakov D1-Brane Action in the presence of a Scalar Axion Field and an $U(1)$ Gauge Field,” Few Body Systems, Vol. 42, No. 1-4, 2011.

[20] P. A. M. Dirac, "Generalized Hamiltonian Dynamics," Canadian Journal of Mathematics, Vol. 2, 1950, pp. 129148. doi:10.4153/CJM-1950-012-1

[21] M. Henneaux and C. Teitleboim, "Quantization of Gauge Systems,” Princeton University Press, Princeton, 1992.

[22] P. Senjanovic, "Path Integral Quantization of Field Theories with Second-Class Constraints," Annals Physics, Vol. 100, No. 1-2, 1976, pp. 227-261. doi:10.1016/0003-4916(76)90062-2

[23] U. Kulshreshtha, "Hamiltonian, Path Integral and BRST Formulations of the Chern-Simons-Higgs Theory in the Broken Symmetry Phase,” Physica Scripta, Vol. 75, No. 6, 2007, pp. 795-802. doi:10.1088/0031-8949/75/6/009

[24] U. Kulshreshtha and D. S. Kulshreshtha, “Gauge-Invariant Reformulation of the Vector Schwinger Model with a Photon Mass Term and Its Hamiltonian, Path Integral and BRST Formulations,” International Journal of Modern Physics, Vol. A22, No. 32, 2007, pp. 6183-6201.

[25] U. Kulshreshtha, "Hamiltonian and BRST Formulations of the Nelsen-Olesen Model," International Journal of Theoretical Physics, Vol. 41, No. 2, 2002, pp. 273-291. doi:10.1023/A:1014058806710

[26] P. A. M. Dirac, "Forms of Relativistic Dynamics," Reviews of Modern Physics, Vol. 21, No. 3, 1949, pp. 392399. doi:10.1103/RevModPhys.21.392

[27] S. J. Brodsky, H. C. Pauli and S. S. Pinsky, "Quantum Chromodynamics and Other Field Theories on the LightCone,” Vol. 301, No. 4-6, 1998, pp. 299-486.

[28] U. Kulshreshtha, "Light-Front Hamiltonian, Path Integral and BRST Formulations of the Nelsen-Olsen (Bogomol'nyi) Model in the Light-Cone Gauges," International Journal of Theoretical Physics, Vol. 46, No. 10, 2007, pp. 2516-2530. doi:10.1007/s10773-007-9367-5

[29] U. Kulshreshtha, D. S. Kulshreshtha and J. P. Vary, "Light-Front Hamiltonian, Path Integral and BRST Formulations of the Chern-Simons-Higgs Theory under Appropriate Gauge-Fixing,” Physics Scripta, Vol. 82, No. 5, 2010, p. 055101. doi:10.1088/0031-8949/82/05/055101

[30] U. Kulshreshtha, D. S. Kulshreshtha and J. P. Vary, "Light-Front Hamiltonian, Path Integral and BRST Formulations of the Chern-Simons Theory under Appropriate Gauge-Fixing,” Journal of Modern Physics, Vol. 1, No. 6, 2010, pp. 385-392.

[31] U. Kulshreshtha, D. S. Kulshreshtha and J. P. Vary, "Light-Front Hamiltonian, Path Integral and BRST For- 
mulations of the Chern-Simons Theory under Appropriate Gauge-Fixing,” Journal of Modern Physics, Vol. 1, No. 6, 2010, pp. 85-392.

[32] U. Kulshreshtha, D. S. Kulshreshtha and J. P. Vary,
"Light-Front Hamiltonian, Path Integral and BRST Formulations of the Chern-Simons-Higgs Theory under Appropriate Gauge-Fixing,” Physics Scripta, Vol. 82, No. 5, 2010, p. 055101. doi:10.1088/0031-8949/82/05/055101 